\title{
VISUALISED FRAMES: HOW SKETCHING INFLUENCES FRAMING BEHAVIOUR IN DESIGN TEAMS
}

\author{
Yang, Yujing; Brik, Natalie; de Jong, Peter; Guerreiro Goncalves, Milene \\ Delft University of Technology
}

\begin{abstract}
Framing is a crucial skill for connecting problem and solution spaces in the creative design process, both for individuals and teams. Frames are implicit in individuals' cognitive thinking, but the creation of shared frames plays a vital role in collaborative design. Many studies have attempted to describe the framing process, but little is still known about how to support designers in framing, specifically in teams. This paper addresses this gap, by exploring the connection between sketching and framing within interdisciplinary teams. Following a qualitative and explorative approach, we have investigated the process and outcome of five interdisciplinary teams. We identified that sketching assists in the creation and elaboration of frames. Furthermore, in tandem with discussion and reflection, sketching helps increase the chance of a frame to survive within the design process. Our findings have practical and educational implications for improving the creative design process in interdisciplinary teams.
\end{abstract}

Keywords: Framing, Sketching, Collaborative design, Design process, Visualisation

\section{Contact:}

Yang, Yujing

TU Delft

Industrial Design Engineering

The Netherlands

Y.Yang-19@student.tudelft.nl 


\section{INTRODUCTION}

The design process is generally accepted to be highly iterative (e.g., Hybs and Gero, 1992). In the same way, creativity does not happen linearly, starting from a well-defined problem, with only one possible correct solution in sight. Instead, design problems are considered to be ill-defined (e.g., Rittel and Webber, 1973), which are interpreted and redefined several times, resulting in both problem and solution spaces co-evolving together until a problem-solution pair is framed (e.g., Maher, Poon and Boulanger, 1996; Dorst and Cross, 2001).

During this co-evolution process over time, frames play an important role in establishing (temporarily) bridges between problem understanding and solutions creation (Dorst and Cross, 2001; Schön, 1983). The ability of (re)framing has been indicated as one of the key strengths of a designer, which is connected to a better understanding of the problem and corresponding solutions (Christiaans, 1992; Dorst, 2011; Pee et al., 2015). Frames are considered to be "underlying structures of belief, perception, and appreciation" (Schön and Rein, 1994, p. 23). Therefore, by definition, frames are implicit and difficult to observe. Prior studies on framing have attempted to uncover such elusive individual and shared frames by extracting them from narratives or teams conversation (e.g., Schön, 1983; Stompff, Smulders and Henze, 2016), but knowledge sharing is just one possible design activity to consider. Co-evolution and the design process is manifested through a large variety of activities, which are idiosyncratic to design (Cash and Gonçalves, 2017; Cash, Hicks, and Culley, 2015) and, arguably, one of the most important design activities is sketching. However, no extensive prior research has investigated the dynamic interaction between framing and sketching in the design process. For instance, Schön (1983) describes the design process as a conversation with the situation. While thinking, writing and sketching, designers are able to reflect on the situation and change it, supported by design representations. Others have helped establish that sketching enables the resolution of conflicting frames, by making them visible and, thus, explicit (Akin, 1978; Hey, Joyce and Beckman, 2007). Despite those general contributions, far too little attention has been given to how actually frames are generated - and not only made explicit - through sketching. Thus, the aim of this study is to establish an understanding of how sketching can support frame creation.

\section{LITERATURE REVIEW}

Starting from Schön's definition of frames (Schön and Rein, 1994), presented in the previous section, frames are diffusely defined. Nevertheless, scholars usually agree that frames are strongly shaped by the individual's experiences and cultural backgrounds (Hey et al., 2007). They determine a lens through which the world is seen or, more specifically, what is considered relevant, according to what goal or criteria (Hey et al., 2007; Pee et al., 2015). Although implicit and subjective by nature, frames are not static, and can also be negotiated, shared and reframed within teams.

Taking this definition of frames forward, we now move to framing - the process of creating frames, which has gathered much interest in recent years (e.g., Dorst, 2015). Yet, the study of frame creation is not yet widely understood and there is no generally accepted understanding of how frames are generated (e.g., Borah, 2011; Paton and Dorst, 2011). This is even more emphasised by the myriad of relevant terms that could be used to describe similar concepts, such as shared understanding (Kleinsmann and Valkenburg, 2008), team mental models (Dong, Kleinsmann and Deken, 2013), or transactive memory (Wegner, 1987).

Inspired by Schön, Valkenburg and Dorst (1998) developed a model that both describes the reflective practice and provides an analysis tool of design activities (Dorst, 1997; Valkenburg, 2000; Valkenburg and Dorst, 1998). As such, the design process is simplified into four activities: naming, framing, moving and reflecting. Iteratively, designers name what is relevant in a situation, and by doing so, boundaries are built, which results in setting a frame. It is within frames that designers act or move, and they can include ideating, prototyping or sketching. Designers reflect not only on their moves but also on how adequate their frames are, which can lead to the creation of new frames. Thus, reframing can be defined as embracing another frame for guiding activities. This model is generally accepted and it has been applied frequently as a coding frame in design protocols (e.g., Self and Pei, 2014; Stompff et al., 2016).

As hinted before, many studies agree that sketches support designers in reinterpreting a situation (Schön, 1983; Christiaans, 1992; Purcell and Gero, 1998; Fish and Scrivener, 1990; Goldschmidt, 
2007). Christiaans (1992) states that sketches represent the abstracted knowledge from a problem and provide designers with new knowledge, which can lead to further re-interpretation (Purcell and Gero, 1998). Likewise, Goldschmidt (2007) proposed that quick sketches in teams are not only a communication tool but also support visual mental models in design. From the perspective of the individual designer, Self and Pei (2014) found that sketching makes it possible to revisit prior frames, which elicits reinterpretation of previous ideas and reframing.

Most of the literature mentioned in this review concerns frames within the practice of individual designers, with the exception of Stompff et al. (2016) and Hey et al. (2007). On the other hand, although a number of studies have suggested the relevance of sketching within framing, there is lack of understanding on how sketching supports the act of framing and reframing within teams. After all, innovation emerges from collaboration. More and more organizations and teams are interested in applying a designerly way approach to solve problems (Stompff et al., 2016) and framing is a necessary capability to do so (Dorst, 2015). However, making frames explicit and creating a shared understanding in a team is challenging (Hey et al., 2007), not only for the teams themselves but also for researchers who study this phenomenon.

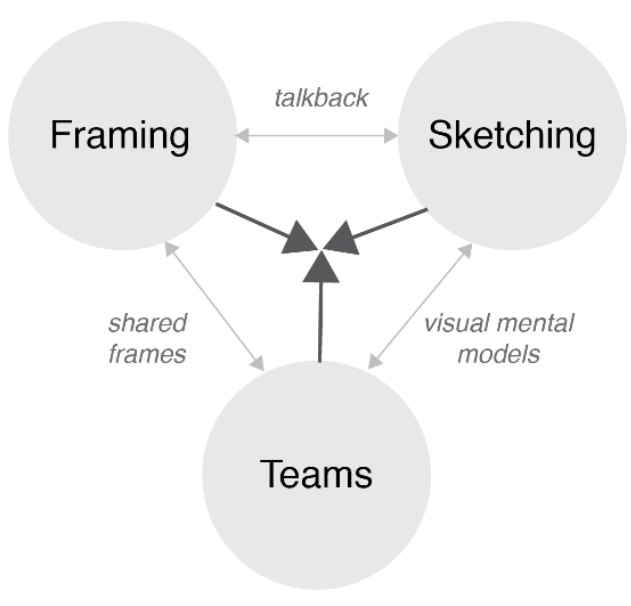

Figure 1. The gap of knowledge addressed in this study lies in the intersection of framing, sketching and design team, represented by the three arrows pointing to the middle

Therefore, this study investigates a triad of concepts: the role of sketching in supporting framing in design teams (Figure 1). This can be translated into the following research question: How does sketching stimulate the creation of frames in the design process of interdisciplinary teams?

By discovering the relation between sketching and framing, we aim to build on the current body of knowledge on these topics. Regarding design practice and education, mastering the framing process could result in better collaboration skills, by improving shared understanding and working in a more goal-oriented manner (Kleinsman et al., 2012). Additionally, teams with a better shared understanding of frames tend to be more successful (Hey et al., 2007).

\section{METHOD}

To deepen the understanding of the relationship between sketching and framing, an empirical and explorative study was conducted, where we followed the work of design teams. Because frames originate from the minds of individuals (Hey et al., 2007), using verbal protocol analysis as a tool provides us with a better understanding of the team's cognitive processes (Suwa, Gero and Purcell, 1998).

\subsection{Data collection}

The study is set up with five interdisciplinary student teams, recruited from a technology university. Following the example of Jiang and Yen's (2009) comparative studies among 134 pieces of research, the protocols of five teams were collected. Each team consisted of four students. The participants were students because, compared with experienced teams, students make less use of jargon, which might facilitate the identification of frames among team members (Stompff et al., 2016). All teams had at least two students from an Industrial Design school and two other members from other 
engineering studies. Holding different backgrounds and vocabularies, participants from different disciplines show distinctive individual frames (Stompff et al., 2016), which may offer more opportunities for them to communicate before a shared frame arises. The participants were sampled purposefully (Patton, 2002, p. 243), to make sure each team includes at least one participant who was comfortable with sketching.

The task of each team was to address the following design problem: As a group, design a solution to help to separate the trash in a student house. It could be a product, service or an integrated solution. This problem was considered to be relatable for all students, and everyone could bring in their individual frames. The teams were given one hour, where they should explore and ideate on the given problem and come up with a final solution. This solution should be communicated in one final poster. To ensure that their self-perceived drawing abilities would not prevent them from representing their ideas, we reinforced that their abilities were not evaluated. Furthermore, we encouraged them to draw during the session.

During the sessions, at least two of the authors were present, to take notes and to audio- and videorecord them. All sketches were collected and used for analysis. The first session, initially planned as a pilot to test its duration and sketching behaviour, was proved to be successful. When analysing the data, we used the pilot study as a regular session.

\subsection{Data analysis}

The five conducted sessions were all transcribed and coded. The coding was focused on framing activities and interaction between participants and sketches. The codes on the framing activities are divided into three categories: 'naming', 'moving' and 'reflecting', loosely based on Valkenburg and Dorst (1998), Valkenburg (2000) and Stompff et al. (2016), as shown in Table 1. To code frames and to add granularity to our analysis, we decided to identify every idea produced by the teams within a bigger concept as a frame. For example, within the concept of an electronic system, the ideas of a 'points system', 'an app' and 'a competition between houses' were considered separate frames.

For coding sketching behaviour, we looked at the activities around sketches, to make it possible to bridge framing with sketching. We coded sketching interacting activities as 'starting' (with sketch), 'pointing' (at sketch), 'adding' (changing sketch) and 'presenting' (sketch), Table 2.

Three authors did the coding, and each of them coded 1 or 2 sessions. To check consistency between different researchers, we took a small part of the sessions and coded separately. The differences in codes were discussed and the coding scheme was refined, but no inter-rater agreement was calculated.

Table 1. Coding table for framing codes

\begin{tabular}{|l|l|}
\hline Code & Definition \\
\hline Naming & Refer to design brief or refer to existing solution \\
\hline Moving & $\begin{array}{l}\text { Sketching, acting, building on other's ideas, sharing thoughts, coming up } \\
\text { with arguments }\end{array}$ \\
\hline Reflecting & Questioning an idea, thinking about consequences, making decisions \\
\hline
\end{tabular}

Table 2. Coding table for interacting codes

\begin{tabular}{|l|l|}
\hline Code & Definition \\
\hline Starting & Starting with a new sketch \\
\hline Showing & Showing sketch to others in the team \\
\hline Pointing & Pointing at a sketch that is already on paper \\
\hline Adding & Adding to an existing sketch (e.g., arrows, colour, words) \\
\hline
\end{tabular}




\section{RESULTS}

From the data analysis, three main insights were derived. Firstly, sketches could act as explicit frames; Secondly, sketches also serve as references for reflecting and to evoke interaction activities; Thirdly, the chance of a frame to be further elaborated on and end up as the final solution is related to both pictorial and verbal interactions combined.

\subsection{Sketches as manifestation of frames}

During the sessions, we identified that individual frames were made explicit through sketching. If and when other team members were enthusiastic about those frames, they often followed similar representations, revealing the adoption of that frame. This seems to indicate that sketches are a manifestation of individual frames, which supports the team to reveal and share individual frames and iterate on each other.

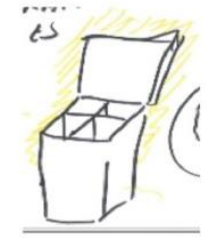

The sketch made by S3_M

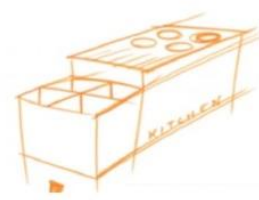

The sketch made by S3_J

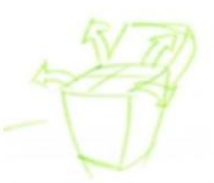

The sketch made by $\mathbf{S} 3 \mathrm{X}$

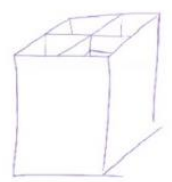

The sketch made by S3_o

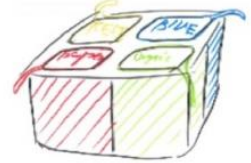

The final sketch made by $\mathbf{S} 3$ _

Figure 2. The first sketch that resulted in drawings made by all participants with a lot of similarity with the first sketch

As an example, in session 3 a participant sketched a bin with four dividers and explained his idea to others (Figure 2). His frame, which could be described as 'garbage division', was accepted by the team and from then on, this frame became prevalent on following sketches. In session 2, we saw a similar example of the frame "Hero bag", sketched differently by many team members (Figure 3).

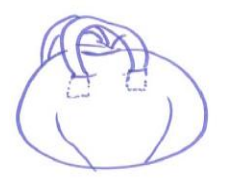

The sketch made by S2_c

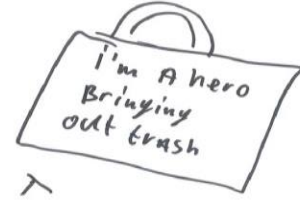

The sketch made by $\mathbf{S 2}$ S

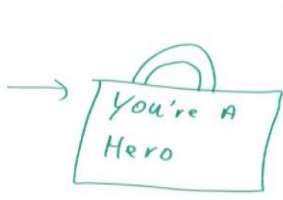

The sketch made by $\mathbf{s 2}$ _c

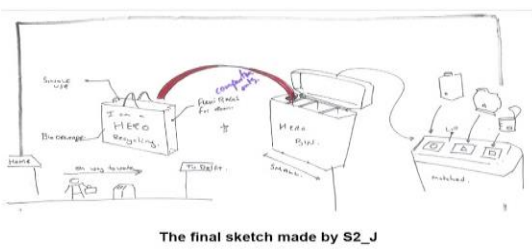

The final sketch made by s2

Figure 3. The iterations of the 'hero bag' sketches, the idea stayed alive and is represented in the final idea

These examples show the importance of sketching for building shared frames. This finding contributes to the previous work of Hey et al. (2007): while they focused on how teams aligned and coordinated activities into a shared and mutual whole, we were able to identify that sketching, as an activity, supports individual frames to become explicit, and later, shared.

\subsection{Sketches provoke reflection through interactions}

A number of studies have established that sketches can support designers in evaluation and idea refining (Ewenstein and Whyte, 2009; Self and Pei, 2014). To add to this, we found that interactions around sketches, which include pointing, circling and adding to the sketches, are also valuable for the process of reflecting and iterating. Figure 4 illustrates this finding. A participant drew a stacked-box-bin idea. The sketch triggered a bunch of discussions in the team immediately. All the participants were interacting with the sketch, by pointing, circling or adding new features on the sketch. The discussion and refinement of this one sketch end up with stimulating one participant to sketch a new idea based on the original sketch.

Sketches offer an accessible database of ideas to refer back to because they enable frames to be manifested and stored. In this way, teams could easily interact with earlier ideas and reinterpret them. Idea representations help to reflect and evaluate the existing frame, which creates the opportunity for a new frame to emerge. 

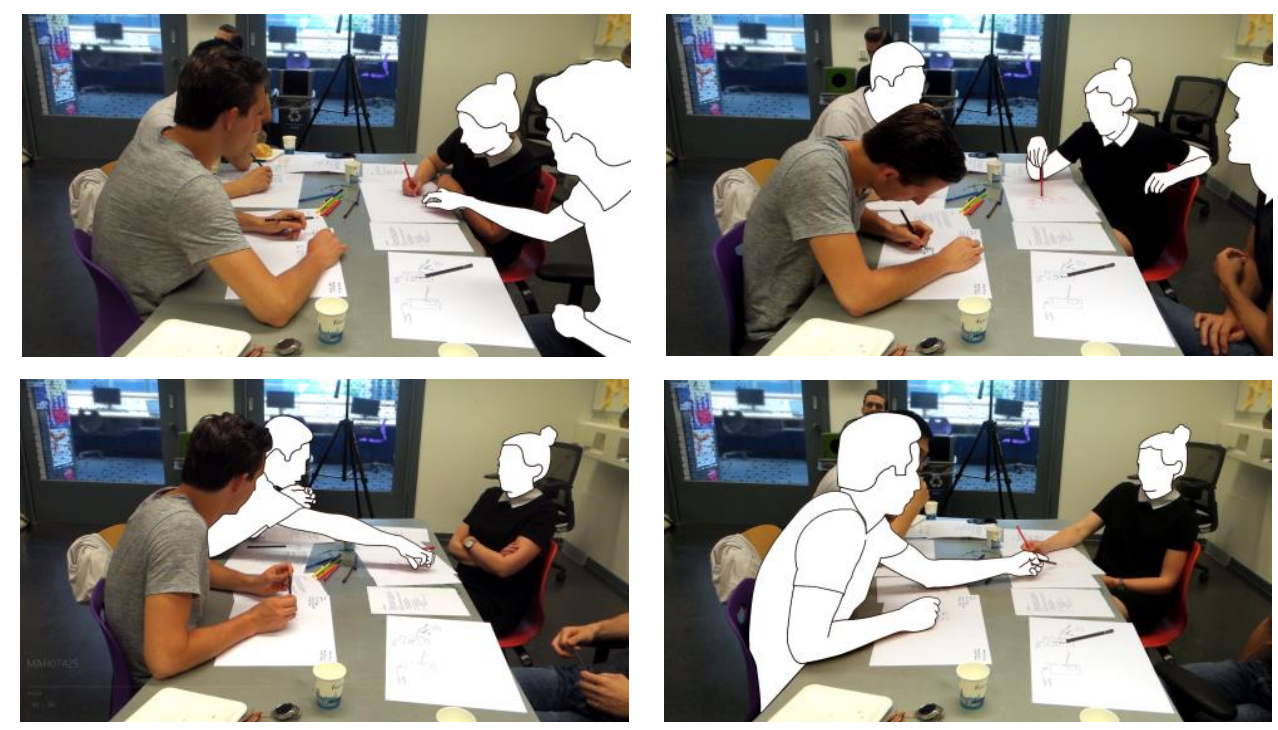

Figure 4. One of the sketches was discussed and iterated upon, supported by interactions around it. This triggered another team member to sketch a new idea. The participants are outlined for anonymisation and to highlight the interaction

\subsection{Sketching supporting the life (or death) of frames}

We have observed that some ideas came back in the discussion often, while other ideas were discarded without being mentioned later. This is closely related to pictorial and verbal activities. When an idea was both sketched and discussed by the team, it invariably led to its elaboration. Conversely, when an idea was only verbally described and not visually represented, the engagement of the rest of the team was lower and the idea was abandoned. A reason behind the identified relationship could be the ability of the group to remember ideas. Visual representations provide stronger distinctive features than words (Humphreys and Bruce, 1989). These features make it easier for the group to recall and follow up on the ideas.

Table 3. Total number of ideas produced in all sessions, classified into three categories: sketching, talking, sketching + talking

\begin{tabular}{|l|c|c|c|r|}
\hline & & Sketching & Talking & Sketching + Talking \\
\hline Session 1 & \# of ideas (total) & 3 & 12 & 14 \\
\hline Session 2 & \# of ideas (survived) & 1 & 2 & 10 \\
\hline & \# of ideas (total) & 4 & 19 & 7 \\
\hline \# of ideas (survived) & 0 & 4 & 17 \\
\hline Session 3 & \# of ideas (total) & 2 & 12 & 10 \\
\hline Session 4 & \# of ideas (survived) & 0 & 1 & 17 \\
\hline \# ideas (total) & \# of ideas (survived) & 0 & 15 & 11 \\
\hline Session 5 & \# ideas (total) & 1 & 0 & 9 \\
\hline & \# of ideas (survived) & 0 & 11 & 4 \\
\hline
\end{tabular}




\begin{tabular}{|l|c|c|c|r|}
\hline All sessions & \# of ideas (total) & 20 & 69 & 70 \\
\hline & \# of ideas (survived) & 2 & 11 & 27 \\
\hline & & $10 \%$ & $16 \%$ & $66 \%$ \\
\hline
\end{tabular}

Furthermore, we identified a relationship between 'talking and sketching activities' and the 'chance to survive' of an idea. 'Chance to survive' is a colloquial expression to indicate that an idea (or its parts), which emerged earlier in a session, end up in the final concept, as presented in a poster. Table 3 and Figure 5 show this relationship. More specifically, if a team member sketched an idea, but did not verbally explain, it has a low chance to survive. Similarly, many ideas that were only discussed (but not put on paper) did not often 'survive' until the final poster.
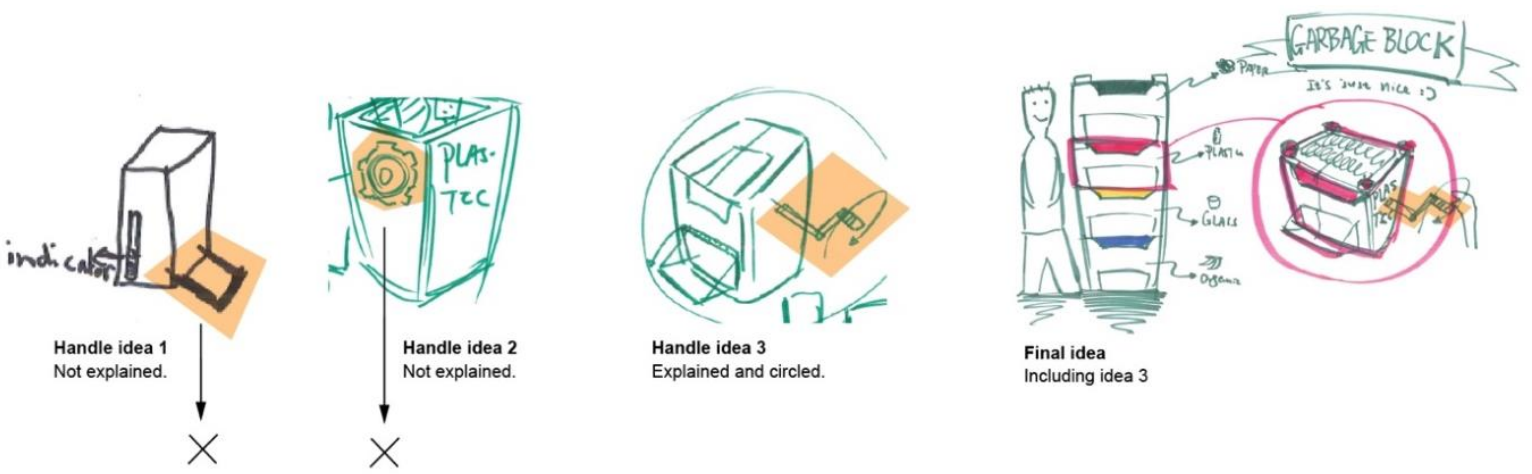

Figure 5. Example of a frame, represented by four different sketches, where the 'handle' part survived till the final poster

Sketches have been indicated as a way to store ideas (Van der Lugt, 2005). Therefore, the life and death of an idea, or its 'chance of survival', could be related to the effort a team is willing to put in storing a preferred idea, but not an idea that is not immediately picked up by the team members.

\section{DISCUSSION}

In this section, we will dive deeper into our findings to answer the initial research question: How does sketching stimulate the creation of frames in the design process of interdisciplinary teams? The main insights are shown in Figure 6 and used as a basis to explain the process of how sketching helps the creation of frames.

\subsection{Sketches as an accessible database to build shared frames}

Through sketching, individual frames are made explicit on paper. As previously shown (Self and Pei, 2014), sketching helps individuals reflect on previous ideas, which is illustrated in Figure 6, with path 1. Prior studies (e.g., Hey et al., 2007) had also already demonstrated that open communication supports the manifestation of frames (path 2). In our study, we additionally found that when teams continuously discuss and interact with sketches, by combining visual and verbal communication, frames become more explicit among team members (path 3). These manifested frames do not need to be immediately used; they can be stored and temporarily put aside (path 4). Afterwards, the team is able to return to stored frames. When being made explicit and manifested, frames can be evaluated, negotiated and further explored. Thus, sketches and talking around sketches facilitate reflection, which is especially important in interdisciplinary teams, to arrive to a shared frame (path 5). Our work expands on previous studies, such as the ones of Purcell and Gero (1998), Hey et al. (2007) or Self and Pei (2014), by elaborating on the team level influence of sketching in reflecting and framing activities.

\subsection{Sketches provoking creation and elaboration of frames}

Through sketching and interactions around it, individual frames are shared explicitly, thus enabling the emergence of a shared frame eventually (path 6 of Figure 6). From that point, the value of a frame is evaluated, negotiated and elaborated by the team, a finding supported also by Hey et al. (2007). The team reflects on whether or not the proposed frame is adequate to guide the subsequent activities. The 
deeper they explore and negotiate the original frames, the more elaborated the frames can become (path 7). Nevertheless, frames can also be discarded, specifically when they do not satisfy the problem interpreted by the team (path 8). In this way, sketches lead to the creation of new frames. These frames could be totally new, or a rearrangement of earlier ideas into a new frame. This rearrangement of frames by use of sketches is consistent with findings from other studies (e.g., Self and Pei, 2014), which have indicated sketching as a stimulus for the re-emergence of new problem frames.

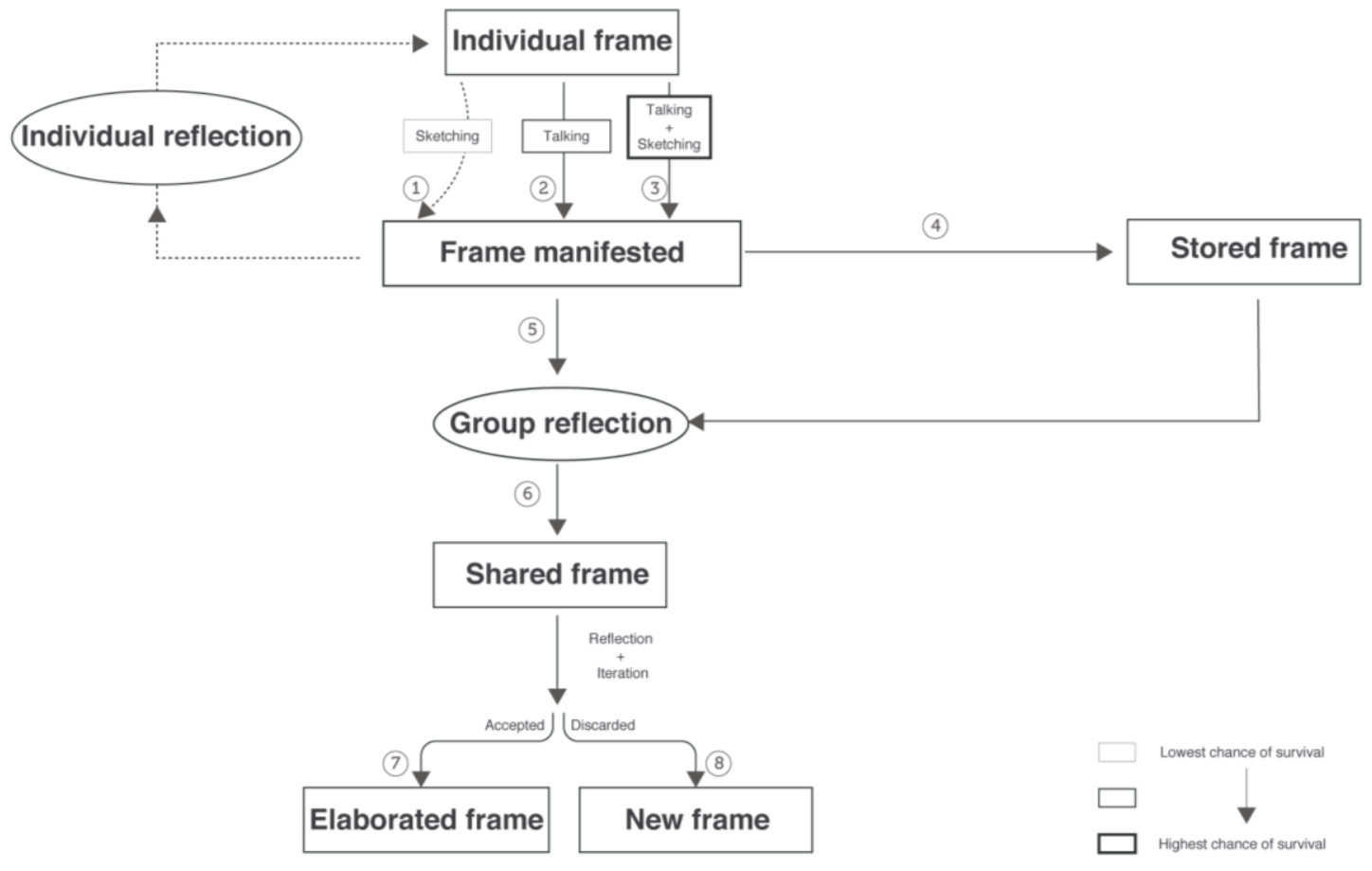

Figure 6. The process of how sketches support framing behaviour

\subsection{Sketching increases the chance of the survival of frames}

As illustrate in Figure 6, a frame could be manifested in three ways (path 1,2 and 3). With only sketching, a frame tends to stay at the individual level. Combined with verbal communication, sketches facilitate shared understandings in teams (e.g., Kleinsmann and Valkenburg, 2008). Arguably, shared frames can also be realized via only verbal communication. However, our findings indicate that when a team is highly involved in discussing and sketching together, ideas have the highest chance to become leading concepts and "survive" the entire design process. Ideas that are made explicit and visualised enable the team to add and change features, which is more challenging only through talking. In contrast, with only sketching, a frame could stay individual and implicit. Thus, frames that are sketched without the support of verbal communication have the lowest chance of survival. Hence, combining sketching and talking - visual and verbal communication - supports frames to become more detailed and increase the chance they are selected at the end. However, this could also make it more difficult to step out of a current undesirable frame, a phenomenon closely related to design fixation and mental set (e.g., Crilly and Cardoso, 2017). According to these authors, "design fixation is a state in which someone engaged in a design task undertakes a restricted exploration of the design space due to an unconscious bias resulting from prior experiences, knowledge or assumptions" (Crilly and Cardoso, 2017, p. 6). Our results indicate that teams tended to adhere to similar sketches for the same frame during the whole session, creating boundaries for their own creativity. We have not specifically addressed design fixation in our analysis, hence we are unable to show whether the teams were at times fixated. Nevertheless, group reflection should contribute to the awareness of one being fixated (Lane and Jensen, 1993; Crilly and Cardoso, 2017). 


\section{CONCLUSION}

This paper investigated the connection between sketching and framing behaviour in the design process of interdisciplinary teams. To answer our research question, we used verbal protocol analysis to study framing behaviour (based on the reflective model proposed by Valkenburg and Dorst, 1998), and interactions around sketching. A number of findings emerged from our analysis: Through reflecting, sketches do provoke the emergence of new frames. Moreover, sketches contribute to the creation of shared frames within the team, by acting as a manifestation of an existing frame. This manifestation of frames on paper enables ideas to be stored and returned to later. Additionally, we also found that the combination of both verbal and visual communication support frames in retaining their importance in the design process for a longer period. This means that if frames are both sketched and discussed upon, there are higher chances that they survive and are selected as final concepts.

A number of possible limitations need to be acknowledged regarding this study. Our findings concern the analysis of 5 interdisciplinary teams, where two design students were present. This could be considered a small sample and it is important to consider this before transferring our findings to a larger context. Therefore, future attention should be given to the continuation of exploring this topic with a larger sample size.

This research is limited by only taking a qualitative perspective on the creation of frames. Comparing sessions with and without sketching could reveal differences in quality and quantity of created frames. Cross and Cross (1998) indicated that more reframing leads to better results, but this was an aspect impossible to explore in this paper, due to the qualitative nature of our study. Future research could compare different set-ups, regarding multiples approaches to sketching, with and without verbal communication to support frame creation. This could shed some light on the relationship between frame creation and the quality and creativity of design outcome. Furthermore, post-interviews after the sessions, focusing on how individuals perceived their own frames, could have resulted in broader insights into the implicit nature of framing. It is then recommended to combine multiple sources of data to corroborate findings retrieved from the already collected verbal protocols and sketches.

In our study, we have not considered individual differences and traits, nor the influence of group dynamics. For instance, it may be that sketching and talking at the same time could be difficult, varying per person; or the chance of survival of an idea might also be related to the dominance of specific team members. Future research could focus on the ability of people to visualise and communicate and how this combination could be of educational relevance.

Finally, this paper has implications for design research, practice and education. A take-away for designers is to reinforce the relevance of visual communication to ensure frame sharing among team members and stakeholders. More importantly though, is that visual communication alone is not enough, it should be combined with discussion and reflection. Team members who both explain and sketch their ideas tend to naturally have more influence on the design process. This influence could nevertheless be undesirable, as it does not mean that those who sketch well are automatically skilled designers. In education, it is important to continue reinforcing the relevance of visualisation (through sketching, prototyping, or other forms) in the design curricula. Those who are less familiar with visualisation should be aware of the fact that they might be jeopardising their success in team settings. To support frame creation, manifestation and sharing among team members, we have demonstrated the importance of visual and verbal communication in tandem can have in interdisciplinary teams.

\section{REFERENCES}

Akin, O. (1978), “How do architects design?", in J.C. Latombe (Ed.), Artificial Intelligence and Pattern Recognition in Computer Aided Design. North Holland Publishing Company, New York, (pp. 65-103).

Borah, P. (2011), "Conceptual issues in framing theory: a systematic examination of a decade's literature", Journal of Communication, Vol. 61, No. 2, pp. 246-263.

Cash, P., Hicks, B. and Culley, S. (2015), "Activity Theory as a means for multi-scale analysis of the engineering design process: A protocol study of design in practice". Design Studies, Vol. 38 No. (May), pp. $1-32$.

Cash, P. and Gonçalves, M. (2017), "Information-triggered Co-evolution: A Combined Process Perspective”. In Christensen, B.T., Ball, L.J. and Halskov, K. (Eds.), Analysing Design Thinking: Studies of Cross-Cultural Co-Creation. CRC Press. (pp. 501-520).

Crilly, N. and Cardoso, C. (2017), "Where next for research on fixation, inspiration and creativity in design?", Design Studies, Vol. 50, pp. 1-38. 
Cross N. and Cross A.C. (1998), "Expert Designers”. In: Frankenberger E., Birkhofer H., Badke-Schaub P. (eds) Designers. Springer, London.

Christiaans, H. (1992), Creativity in design: the role of domain knowledge in designing. Ph.D. Thesis, Delft University of Technology, Delft, The Netherlands. (pp. 145-150).

Dong, A., Kleinsmann, M. and Deken, F. (2015), "Investigating design cognition in the construction and enactment of team mental models". Design Studies, Vol. 34, No. 1, pp. 1-33.

Dorst, K. (1997), Describing Design, a Comparison of Paradigms. Ph.D. thesis. Delft University of Technology, Delft, The Netherlands.

Dorst, K. and Cross, N. (2001), “Creativity in the design process: co-evolution of problem-solution”. Design studies, Vol. 22, No. 5, pp. 425-437.

Dorst, K. (2011), “The core of 'design thinking' and its application”. Design Studies, Vol. 32, No. 6, pp. 521532.

Dorst, K. (2015), "Frame creation and design in the expanded field". She Ji: The Journal of Design, Economics, and Innovation, Vol. 1, No. 1, pp. 22-33.

Fish, J. and Scrivener, S. (1990), “Amplifying the mind's eye: sketching and visual cognition”. Leonardo, pp. $117-126$.

Goldschmidt, G. (2007), "To see eye to eye: the role of visual representations in building shared mental models in design teams", CoDesign, Vol. 3, No. 1, pp. 43-50.

Hey, J.H., Joyce, C.K. and Beckman, S.L. (2007), "Framing innovation: negotiating shared frames during early design phases". Journal of Design Research, Vol. 6, No. 1-2, pp. 79-99.

Hybs, I. and Gero, J. (1992), “An evolutionary process model of design”. Design Studies, Vol. 13, No. 3, pp. 273-290.

Jiang, H. and Yen, C.C. (2009), "Protocol Analysis in Design Research: a review". In Design |Rigor \& Relevance”, International Association of Societies of Design Research (IASDR) 2009, Seoul, Korea.

Kleinsmann, M., Deken, F., Dong, A. and Lauche, K. (2012), "Development of design collaboration skills". Journal of Engineering Design, Vol. 23, No. 7, pp. 485-506.

Lane, D.M. and Jensen, D.G. (1993), "Einstellung: Knowledge of the Phenomenon Facilitates Problem Solving". Proceedings of the Human Factors and Ergonomics Society Annual Meeting, Vol. 37, No. 18, pp. 12771280.

Maher, M.L., Poon, J. and Boulanger, S. (1996), "Formalising design exploration as co-evolution". In Advances in formal design methods for CAD. Springer, Boston, MA. (pp. 3-30).

Paton, B. and Dorst, K. (2011), "Briefing and reframing: A situated practice". Design Studies, Vol. 32, No. 6, pp. 573-587.

Patton, M.Q. (2002), “Two decades of developments in qualitative inquiry: A personal, experiential perspective". Qualitative social work, Vol. 1, No. 3, pp. 261-283.

Pee, M., Dorst, K. and van der Bijl-Brouwer, M. (2015), "Understanding Problem Framing through research into Metaphors". In IASDR 2015 Conference.

Purcell, A. and Gero, J.S. (1998), "Drawings and the design process: A review of protocol studies in design and other disciplines and related research in cognitive psychology". Design studies, Vol. 19, No. 4, pp. 389430.

Rittel, H.W. and Webber, M. (1973), "Dilemmas in a general theory of planning”. Policy Sciences, Vol. 4, No. 2, pp. $155-169$

Schön, D.A. (1983), The reflective practitioner: how professionals think in action (Vol. 1). Basic Books, NY.

Schön D.A. and Rein M. (1994), Frame Reflection: Toward the Resolution of Intractable Policy Controversies. Basic Books, New York.

Self, J. and Pei, E. (2014), "Reflecting on Design Sketching: Implications for Problem-Framing and Solution focused Conceptual Ideation". Archives of Design Research, Vol. 27, No. 3, pp. 65-87.

Self, J.A. (2016), "Problem or solution focused? Ill-defined design problems and the influence of design ability". In DS 84: Proceedings of the DESIGN 2016 14th International Design Conference.

Stompff, G., Smulders, F. and Henze, L. (2016), "Surprises are the benefits: reframing in multidisciplinary design teams". Design Studies, Vol. 47, pp. 187-214.

Suwa, M., Purcell, T. and Gero, J. (1998), "Macroscopic analysis of design processes based on a scheme for coding designers' cognitive actions”. Design studies, Vol. 19, No. 4, pp. 455-483.

Valkenburg, R. (2000), The Reflective Practice in Product Design Teams. Ph.D. thesis. Delft University of Technology. Delft, the Netherlands.

Valkenburg, R. and Dorst, K. (1998), “The reflective practice of design teams”. Design studies, Vol. 19, No. 3, pp. 249-271.

Van der Lugt, R. (2005), "How sketching can affect the idea generation process in design group meetings". Design studies, Vol. 26, No. 2, pp. 101-122.

Wegner, D.M. (1987), “Transactive memory: a contemporary analysis of the group mind”. In B. Mullen and G. Goethals (eds)., Theories of group behavior, Springer-Verlag, New York, pp. 185-208. 